\title{
Underprivileged in Plymouth receive dental care
}

As the clinical arm of Peninsula Dental School at the University of Plymouth, Peninsula Dental Social Enterprise (PDSE) aims to ensure access to dental care for all, especially those excluded from mainstream dentistry.

PDSE leads community engagement activities within the dental curriculum and beyond, and one of its four clinics is located in one of Plymouth's most disadvantaged areas.

In an effort to help a particularly underprivileged group, and as a pro bono contribution to its social impact, PDSE has provided urgent care and follow-up treatment by a qualified dentist to people who experience

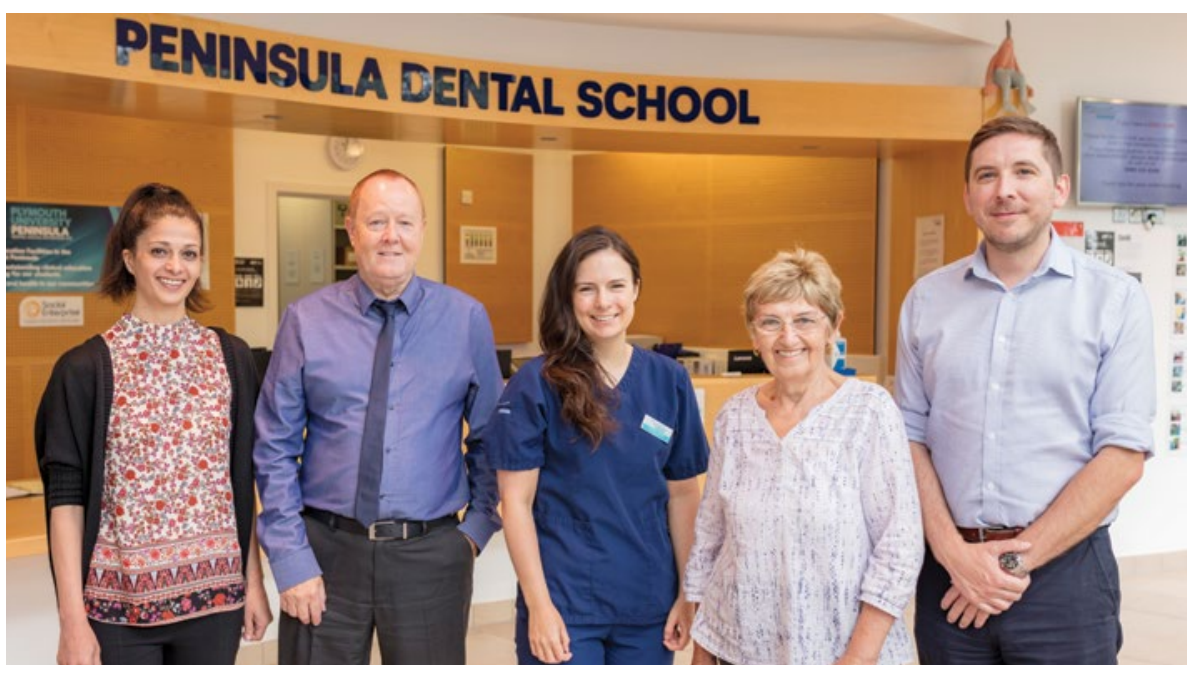

(I-r) Martha Paisi, Colin Massey, Christina Worle, Lyndsey Withers and Rob Witton homelessness. The clinic started in January 2018 for half a day per week, and, due to demand and success, has increased to a full day per week since August 2018.

The service is continuously evolving, based on existing literature, primary research, and the growing experience of PDSE staff in working with homeless patients. By the beginning of July 2019, 61 patients had been seen, with 28 completing a treatment pathway. Evaluation shows that treatment helps patients in terms of pain relief, functionality and motivation to look after their teeth. Moreover, it impacts positively on their confidence, self-esteem and self-worth.

One patient who received treatment commented: 'It's helped me get a job, it's helped me move forward in society ... it's meant a great deal towards my future.'

The clinic is an important public health offer and a valuable learning opportunity for PDSE. A key lesson is the importance of close collaboration with a residential homelessness centre and active communication between the dentist and a centre volunteer.

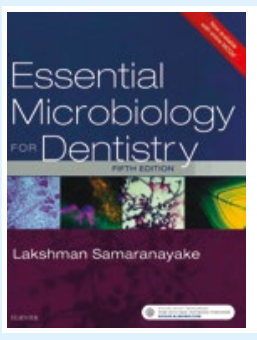

\section{ESSENTIAL MICROBIOLOGY FOR DENTISTRY, FIFTH EDITION}

Lakshman Samaranayake;

2018;

Elsevier;

price f54.99; pp. 400

ISBN: 9780702074356

The Essential microbiology for dentistry textbook continues to flourish as one of the key resources on the topic across the world. With continuing developments in the technology and science of microbiology, this textbook places itself at the forefront by blending the latest research with the essentials from previous editions, to provide exceptional detail for both students and practitioners to learn.

Now entering its fifth edition, the author Lakshman Samaranayake has used his wealth of experience to produce an evidence-based book that relates to modern practice throughout. This edition also has an online bonus resource of 300 MCQs written by Samaranayake himself, excellent for testing knowledge.
The book is divided into six main parts. The first two are general microbiology and basic immunology; these provide a solid foundation on the structure of bacteria and viruses, how they cause disease and how the body responds to an infection by mounting an immune response. Well labelled diagrams, tables and scanning electron micrographs really help to bring this subject to life.

The next four parts are more dentally-focused: microbes and infections of relevance to dentistry; oral microbiology; and cross-infection in practice. Systematic explanations of each relevant microbe provides great continuity for revision. The book also runs through the habitat, characteristics, cultures, pathogenicity and treatment for each.

One of the author's main research interests lies in candida biofilm biology and oral candidiasis. Hence the sections on fungi are exquisitely presented in every aspect. The topical debate of infective endocarditis prophylaxis is raised, with great discussion of both the British and American guidelines. Although these were very accurate at the time of print, care must be taken as updates and changes to the guidance are to be expected. The two more common diseases of dental caries and periodontal disease also have a magnitude of information presented. An interesting section is also included on the postulation of oral-systemic links, where great research is trying to prove the connectivity of oral flora to adverse pregnancy outcomes. The book's conclusion on infection control procedures in dentistry reminds us as clinicians that we must practise the gold standards to ensure the protection of patients and ourselves.

Key facts, review questions and further reading at the end of each part cumulate to produce a perfect study guide and also a great reference for all.

Patrick Steed 